Kragujevac Journal of Mathematics

Volume 45(2) (2021), Pages 259-265.

\title{
SOME REMARKS ON DIFFERENTIAL IDENTITIES IN RINGS
}

\author{
MOHD ARIF RAZA ${ }^{1}$, HUSAIN ALHAZMI ${ }^{1}$, AND SHAKIR ALI ${ }^{2}$
}

\begin{abstract}
Let $1<k$ and $m, k \in \mathbb{Z}^{+}$. In this manuscript, we analyse the action of (semi)-prime rings satisfying certain differential identities on some suitable subset of rings. To be more specific, we discuss the behaviour of the semiprime ring $\mathcal{R}$ satisfying the differential identities $\left(\left[d\left([s, t]_{m}\right),[s, t]_{m}\right]\right)^{k}=\left[d\left([s, t]_{m}\right),[s, t]_{m}\right]$ for every $s, t \in \mathcal{R}$.
\end{abstract}

\section{Motivation}

The work of this manuscript is motivated by the various results established by many well known algebraists (see $[1,2,4,6,7,9-11,14,15,17,19]$, and references therein). The famous and classical result in this direction is due to Jacobson [12]. The theorem to which we want to mention is the following: "any ring in which $s^{k}=s, 1<k \in \mathbb{Z}^{+}$ is necessarily commutative". The above mentioned result generalizes Wedderburn theorem, i.e., "every finite division ring is commutative", and also the result that "any Boolean ring is a commutative ring".

In [10], Herstein discussed the commutativity of a ring and he established that " $a$ ring must be commutative if it satisfies $[s, t]^{n}=[s, t]$, for every $s, t \in \mathcal{R}$, where $1<n \in \mathbb{Z}^{+}$". In 2011, Huang [11] proved that "if a prime ring $\mathcal{R}$ admits a derivation $d$ such that $d([s, t])^{k}=[s, t]_{n}$ for all $s, t \in I$, a nonzero ideal of $\mathcal{R}$ (where $\left.1<k, n \in \mathbb{Z}^{+}\right) R$ is commutative". In 2017, De Filippis et al. [8] proved the following "let $\mathcal{R}$ be a prime ring of characteristic different from 2 , $d$ be a nonzero derivation of $\mathcal{R}, f\left(x_{1}, \ldots, x_{n}\right)$ be a multilinear polynomial over $\mathcal{C}$ and $1<k \in \mathbb{Z}^{+}$ such that $\left(\left[d\left(f\left(r_{1}, \ldots, r_{n}\right)\right), f\left(r_{1}, \ldots, r_{n}\right)\right]\right)^{k}=\left[d\left(f\left(r_{1}, \ldots, r_{n}\right)\right), f\left(r_{1}, \ldots, r_{n}\right)\right]$ for all

Key words and phrases. (Semi)-prime ring, derivation, Engel polynomial, maximal right ring of quotients, generalized polynomial identity (GPI).

2010 Mathematics Subject Classification. Primary: 16W25. Secondary: 16N60, 16 U80.

DOI $10.46793 / \mathrm{KgJMat} 2102.259 \mathrm{R}$

Received: September 27, 2018.

Accepted: November 29, 2018. 
$r_{1}, \ldots, r_{n} \in \mathcal{R}$. Then $f\left(x_{1}, \ldots, x_{n}\right)$ is central valued on $\mathcal{R}$ ". In particular, if multilinear polynomial replace by commutator, then we conclude with the commutativity of rings. On the other hand, Giambruno et al. [9] generalizes Herstein result for Engel polynomial and they established that "a ring must be commutative if it satisfies $\left([s, t]_{m}\right)^{n}=[s, t]_{m}$ ". In view of Giambruno et al. result Raza and Rehman [18] proved that "a prime ring $R$ is commutative if it satisfies $d\left([s, t]_{m}\right)^{n}=[s, t]_{m}$ for all $s, t \in I$, a nonzero ideal of $\mathcal{R}$ ".

In the prospect of above motivation, our intention is to explore the action of prime and semiprime rings satisfying Engel polynomials, which are not multilinear associated with derivations. To be more specific, we discuss the behaviour of the semiprime ring $\mathcal{R}$ satisfying the differential identities $\left(\left[d\left([s, t]_{m}\right),[s, t]_{m}\right]\right)^{k}=\left[d\left([s, t]_{m}\right),[s, t]_{m}\right]$ for every $s, t \in \mathcal{R}$.

\section{The Results}

We use the following notations and definitions, unless otherwise mention, $\mathcal{R}$ be a ring, $Z(\mathcal{R})$ be the center of $\mathcal{R}, \mathcal{Q}$ be a Martindale quotient ring of $\mathcal{R}, \mathcal{U}$ be a Utumi quotient ring of $\mathcal{R}$, and $\mathcal{C}$ be the extended centroid of $\mathcal{R}$ (see [3] for further details). A ring $\mathcal{R}$ is said to be prime if for any $s, t \in \mathcal{R}, s \mathcal{R} t=(0)$ implies $s=0$ or $t=0$, and $\mathcal{R}$ is semiprime if for any $s \in \mathcal{R}, s \mathcal{R} s=(0)$ implies $s=0$. An additive mapping $d: \mathcal{R} \longrightarrow \mathcal{R}$ is said to be a derivation if it satisfies $d(s t)=d(s) t+s d(t)$ for every $s, t \in \mathcal{R}$. If for any fixed $p \in \mathcal{R}, d(s)=[p, s]$, for every $s \in \mathcal{R}$, then $d$ is said to be inner derivation. Moreover, $d$ is said to be $Q$-inner if the extension of $d$ to $Q$ is inner otherwise Q-outer.

We will proceed by first proving the following auxiliary result.

Lemma 2.1. Let $1<k \in \mathbb{Z}^{+}$and $1 \leq m \in \mathbb{Z}^{+}$. Next, $\mathcal{R}=\mathcal{M}_{m}(\mathcal{C})$ be the ring of $m \times m$ matrices over the field $\mathcal{C}$ such that $\left[\left[p,[s, t]_{m}\right],[s, t]_{m}\right]^{k}=\left[\left[p,[s, t]_{m}\right],[s, t]_{m}\right]$. Then $p \in Z(\mathcal{R})$.

Proof. Let $p=\sum_{i j} p_{i j} e_{i j}$, where $e_{i j}$ denotes the usual unit matrix with 1 in $(i, j)$-entry and zero elsewhere and $p_{i j} \in \mathcal{C}$. We show that $p$ is a diagonal matrix. Next, let $s=e_{i j}$ and $t=e_{j j}$ and in view of our hypothesis, we deduce that $\left(\left[p, e_{i j}\right]_{2}\right)^{k}=\left[p, e_{i j}\right]_{2}$, i.e, $-2 e_{i j} q e_{i j}=0$ and hence $p_{j i}=0$ for any $i \neq j$. Therefore, $p$ is a diagonal matrix.

Further, we see that

$$
\left(\left[\left[\varphi(p),[s, t]_{m}\right]\right]_{2}\right)^{k}=\left[\left[\varphi(p),[s, t]_{m}\right]\right]_{2}
$$

is a generalized polynomial identity of $\mathcal{R}$ for $\varphi \in \operatorname{Aut}_{\mathfrak{C}}(\mathcal{R})$. This shows that $\varphi(p)$ is diagonal matrix. Precisely, we consider the automorphism $\varphi(p)=\left(1+e_{i j}\right) p\left(1-e_{i j}\right)$ for any $i \neq j$ and say $\varphi(p)=\sum_{i j} p_{i j}^{\prime} e_{i j}$, where $p_{i j}^{\prime} \in \mathcal{C}$. Since $p_{i j}^{\prime}=0$, then by easy computation we obtain $0=p_{i j}^{\prime}=p_{j j}-p_{i i}$. So, that $p_{j j}=p_{i i}$ holds for any $i \neq j$. This implies that $p \in Z(\mathcal{R})$. This completes the proof. 
Lemma 2.2. Let $1<k \in \mathbb{Z}^{+}$and $1 \leq m \in \mathbb{Z}^{+}$. Next, let $\mathcal{R}$ be a non-commutative prime ring of characteristic different from 2 . If $p \in \mathcal{Q}$ and $\left[\left[p,[s, t]_{m}\right],[s, t]_{m}\right]^{k}=$ $\left[\left[p,[s, t]_{m}\right],[s, t]_{m}\right]$ be a generalized polynomial identity for $\mathcal{R}$, then $p \in \mathcal{C}$.

Proof. We prove this lemma by contradiction, i.e., we assume that $p \notin \mathcal{C}$. Clearly, $\mathcal{Q}$ satisfies $\left[\left[p,[s, t]_{m}\right],[s, t]_{m}\right]^{k}=\left[\left[p,[s, t]_{m}\right],[s, t]_{m}\right]$ (see $[5]$ ). Specifically, as $p \notin \mathcal{C}$ then the above identity is a non-trivial generalized polynomial identity for $\mathcal{Q}$. Thus, $\mathcal{Q}$ is a primitive ring which is isomorphic to a dense ring of linear transformations of a vector space $\mathcal{V}$ over $\mathcal{C}$ (by Martindale's theorem in [16]).

Now firstly we discuss the case when $\operatorname{dim}_{\mathcal{C}}(\mathcal{V})=m$, where $m>1$, a finite positive integer. In this case, the contradiction follows by Lemma 2.1.

We now assume that $\operatorname{dim}_{\mathcal{C}} \mathcal{V}=\infty$. Then, we have

$$
\left(\left[p,[s, t]_{m}\right]_{2}\right)^{k}=\left[p,[s, t]_{m}\right]_{2}, \quad \text { for all } s, t \in \mathcal{Q} .
$$

Moreover, again by Martindale's theorem [16], it follows that $\operatorname{soc}(\mathcal{Q})=\mathcal{H} \neq(0)$ and $e \mathcal{H} e$ is a finite dimensional simple central algebra over $\mathcal{C}$, for any minimal idempotent element $e \in \mathcal{H}$. Moreover, we may assume that $\mathcal{H}$ is non-commutative, otherwise $Q$ must be commutative. Of course, $\mathcal{H}$ satisfies $\left(\left[p,[s, t]_{m}\right]_{2}\right)^{k}=\left[p,[s, t]_{m}\right]_{2}$ (see for example proof of [14, Theorem 1]). As $\mathcal{H}$ is a simple ring, either $\mathcal{H}$ does not contain any non-trivial idempotent element or $\mathcal{H}$ is generated by its idempotents. In this last case, suppose that $\mathcal{H}$ contains two minimal orthogonal idempotent elements $e$ and $f$. By the hypothesis, for $[s, t]_{m}=[e s, f]_{m}=e s f$, we have

$$
\operatorname{exf}(p) \operatorname{exf}=0
$$

in case we get fpesfpesfpe $=0$, by the primeness of $\mathcal{R}$, we get $f p e=0$, where $e$ and $f$ are orthogonal idempotent element of rank 1 . Specifically, as $e$ of rank 1 , we have $e p(1-e)=0$ and $(1-e) p e=0$, i.e., $e p=e p e=p e$. Therefore, $[p, e]=0$ and $[p, \mathcal{H}]=0$, where $\mathcal{H}$ is generated by these idempotent elements. This argument gives the conclusion that $p \in \mathcal{C}$ or $\mathcal{R}$ is commutative. In this last case, we conclude with contradiction.

Thus we take the case when $\mathcal{H}$ cannot contain two minimal orthogonal idempotent elements and so, $\mathcal{H}=\mathcal{D}$ for a suitable division ring $\mathcal{D}$ finite dimensional over its center. This implies that $\mathcal{Q}=\mathcal{H}$ and $p \in \mathcal{H}$. By [20, Theorem 2.3.29 ] ([14, Lemma 2]), $\mathcal{H} \subseteq M_{n}(K), M_{n}(K)$ satisfies $\left(\left[p,[s, t]_{m}\right]_{2}\right)^{k}=\left[p,[s, t]_{m}\right]_{2}$, where $K$ is a field. If $n=1$, then $\mathcal{H} \subseteq F$, a contradiction. Moreover, if $n \geq 2$, then $p \in Z\left(\mathcal{M}_{n}(F)\right)$, as we have just seen.

Finally, consider if $\mathcal{H}$ does not contain any non-trivial idempotent element, then $\mathcal{H}$ is finite dimensional division algebra over $\mathcal{C}$ and $p \in \mathcal{H}=\mathcal{R} \mathcal{C}=\mathcal{Q}$. If $\mathcal{C}$ is finite, then $\mathcal{H}$ is finite division ring, that is, $\mathcal{H}$ is a commutative field and so $\mathcal{R}$ is commutative too. If $\mathcal{C}$ is infinite, then $\mathcal{H} \otimes_{\mathfrak{e}} K \cong M_{n}(K)$, where $K$ is a splitting field of $\mathcal{H}$. In this case, we get the conclusion by Lemma 2.1. 
Theorem 2.1. Let $1<k \in \mathbb{Z}^{+}$and $1 \leq m \in \mathbb{Z}^{+}$. Next, let $\mathcal{R}$ be a prime ring of characteristic different from 2 and $I$ be a nonzero ideal of $\mathcal{R}$. If $I$ satisfies $\left[d\left([s, t]_{m}\right),[s, t]_{m}\right]^{k}=\left[d\left([s, t]_{m}\right),[s, t]_{m}\right]$ for all $s, t \in I$, then $\mathcal{R}$ is commutative.

Proof. We suppose on contrary that $\mathcal{R}$ is non-commutative. Then, by Lemma 2.1 and Lemma 2.2, we discuss the case when $d$ is not Q-inner. Note that

$$
\begin{aligned}
d\left([s, t]_{m}\right)= & \left.\sum_{m=1}^{k}(-1)^{m}\left(\begin{array}{c}
k \\
m
\end{array}\right) \sum_{i+j=m-1} t^{i} d(t) t^{j}\right) x y^{k-m} \\
& +\sum_{m=0}^{k}(-1)^{m}\left(\begin{array}{c}
k \\
m
\end{array}\right) t^{m} d(s) t^{k-m} \\
& +\sum_{m=0}^{k-1}(-1)^{m}\left(\begin{array}{c}
k \\
m
\end{array}\right) t^{m} s\left(\sum_{r+s=k-m-1} t^{r} d(t) t^{s}\right) .
\end{aligned}
$$

Using the hypothesis and well known results, we can say that $\mathcal{R}$ satisfies

$$
\left[d\left([s, t]_{m}\right),[s, t]_{m}\right]^{k}=\left[d\left([s, t]_{m}\right),[s, t]_{m}\right] .
$$

Therefore, we obtain

$$
\begin{aligned}
& \left(\left[\sum_{m=1}^{k}(-1)^{m}\left(\begin{array}{c}
k \\
m
\end{array}\right) \sum_{i+j=m-1} t^{i} d(t) t^{j}\right) s t^{k-m}+\sum_{m=0}^{k}(-1)^{m}\left(\begin{array}{c}
k \\
m
\end{array}\right) t^{m} d(s) t^{k-m}\right. \\
& \left.\left.+\sum_{m=0}^{k-1}(-1)^{m}\left(\begin{array}{c}
k \\
m
\end{array}\right) t^{m} s\left(\sum_{r+s=k-m-1} t^{r} d(t) t^{s}\right), \sum_{m=0}^{k}(-1)^{m}\left(\begin{array}{c}
k \\
m
\end{array}\right) t^{k} x y^{k-m}\right]\right)^{k} \\
= & {\left[\sum_{m=1}^{k}(-1)^{m}\left(\begin{array}{c}
k \\
m
\end{array}\right) \sum_{i+j=m-1} t^{i} d(t) t^{j}\right) s t^{k-m}+\sum_{m=0}^{k}(-1)^{m}\left(\begin{array}{c}
k \\
m
\end{array}\right) t^{m} d(s) t^{k-m} } \\
& \left.+\sum_{m=0}^{k-1}(-1)^{m}\left(\begin{array}{c}
k \\
m
\end{array}\right) t^{m} s\left(\sum_{r+s=k-m-1} t^{r} d(t) t^{s}\right), \sum_{m=0}^{k}(-1)^{m}\left(\begin{array}{c}
k \\
m
\end{array}\right) t^{k} s t^{k-m}\right] .
\end{aligned}
$$

In view of Kharchenko's theorem [13], we get

$$
\begin{aligned}
& \left(\left[\sum_{m=1}^{k}(-1)^{m}\left(\begin{array}{c}
k \\
m
\end{array}\right) \sum_{i+j=m-1} t^{i} w t^{j}\right) s t^{k-m}+\sum_{m=0}^{k}(-1)^{m}\left(\begin{array}{c}
k \\
m
\end{array}\right) t^{m} z t^{k-m}\right. \\
& \left.\left.+\sum_{m=0}^{k-1}(-1)^{m}\left(\begin{array}{c}
k \\
m
\end{array}\right) t^{m} s \sum_{r+s=k-m-1} t^{r} w t^{s}, \sum_{m=0}^{k}(-1)^{m}\left(\begin{array}{c}
k \\
m
\end{array}\right) t^{k} s t^{k-m}\right]\right)^{k} \\
= & {\left[\sum_{m=1}^{k}(-1)^{m}\left(\begin{array}{c}
k \\
m
\end{array}\right) \sum_{i+j=m-1} t^{i} w t^{j}\right) s t^{k-m}+\sum_{m=0}^{k}(-1)^{m}\left(\begin{array}{c}
k \\
m
\end{array}\right) t^{m} z t^{k-m} } \\
& \left.+\sum_{m=0}^{k-1}(-1)^{m}\left(\begin{array}{c}
k \\
m
\end{array}\right) t^{m} \sum_{r+s=k-m-1} t^{r} w t^{s}, \sum_{m=0}^{k}(-1)^{m}\left(\begin{array}{c}
k \\
m
\end{array}\right) t^{k} s t^{k-m}\right],
\end{aligned}
$$


for all $s, t, z, w \in \mathcal{R}$ and hence it satisfied by $\mathcal{Q}$ [3, Theorem 6.4.4]. Thus, $\mathcal{Q}$ is noncommutative as $\mathcal{R}$. Let us take $p^{\prime} \in \mathcal{Q}$ with $p^{\prime} \notin \mathcal{C}$. Also, we can see that $d^{\prime}: \mathcal{Q} \rightarrow \mathcal{Q}$ is a nonzero derivation of $\mathcal{Q}$ defined by $d^{\prime}(s)=\left[p^{\prime}, s\right]$ for all $s \in \mathcal{Q}$. Replacing $z, w$ by $d^{\prime}(s), d^{\prime}(t)$ in the last identity and using (2.3), we obtain that

$$
\left[d^{\prime}\left([s, t]_{m}\right),[s, t]_{m}\right]^{k}=\left[d^{\prime}\left([s, t]_{m}\right),[s, t]_{m}\right],
$$

for all $s, t \in \mathcal{Q}$. Thus, we can write

$$
\left[\left[p^{\prime},[s, t]_{m}\right],[s, t]_{m}\right]^{k}=\left[\left[p^{\prime},[s, t]_{m}\right],[s, t]_{m}\right],
$$

for all $s, t \in \mathcal{R}$. Application of Lemma 2.2 yields the desire conclusion.

As the immediate consequences of the above theorem, we obtain the following results.

Corollary 2.1. Let $1<k \in \mathbb{Z}^{+}$and $1 \leq m \in \mathbb{Z}^{+}$. Next, let $\mathcal{R}$ be a prime ring of characteristic different from 2 and $d$ be a derivation of $\mathcal{R}$ such that $\left[d\left([s, t]_{m}\right),[s, t]_{m}\right]^{k}=$ $\left[d\left([s, t]_{m}\right),[s, t]_{m}\right]$ for all $s, t \in \mathcal{R}$. Then, $\mathcal{R}$ is commutative.

Corollary 2.2. Let $1<k \in \mathbb{Z}^{+}$. Next, let $\mathcal{R}$ be a prime ring of characteristic different from 2 , $d$ be a derivation and $L$ be a noncentral Lie ideal of $\mathcal{R}$. If $\mathcal{R}$ satisfies $[d(u), u]^{k}=[d(u), u]$ for all $u \in L$, then $\mathcal{R}$ is commutative.

Now, we discuss the our last result for semiprime case. We set out with few preliminary notions which are required for the establishment of the proof of our main theorem. More or less of these notions are classical and we introduce them briefly, let $\mathcal{R}$ be a semiprime ring and $\mathcal{C}$ be the extended centroid $\mathcal{R}$. Also, the orthogonal completion of $\mathcal{R}$ is the intersection of all orthogonally complete subset of $\mathcal{Q}$ containing $\mathcal{R}$ and is denoted by $\mathcal{A}=\mathcal{O}(\mathcal{R})$. In [3, Theorem 3.2.7], Beidar et al. proved that " if $M \in \operatorname{spec}(\mathcal{B})$, then $\mathcal{R}_{M}=\mathcal{R} / \mathcal{R} M$ is prime, where $\mathcal{B}=\mathcal{B}(\mathcal{C})$ is a Boolean ring of $\mathcal{C}$ and $\operatorname{spec}(\mathcal{B})$ is the set of all maximal ideal of $\mathcal{B}$ ". We use the notations $\Omega$ - $\Delta$-ring, Horn formulas and Hereditary formulas. For more definitions and related results see ([3], pages 37, 38, 43, 120). Also we use the results obtained by Beidar et el. [3, Proposition 2.5.1 and Theorem 3.2.18] which state that "any derivation $d$ of a semiprime ring $\mathcal{R}$ can be uniquely extended to a derivation of $\mathcal{U}$ (we shall let d also denote its extension to $\mathcal{U})$ " and "let $\mathcal{R}$ be an orthogonally complete $\Omega$ - $\Delta$-ring with extended centroid $\mathcal{C}, \Theta_{i}\left(s_{1}, s_{2}, \ldots, s_{n}\right)$ Horn formulas of signature of $\Omega-\Delta, i=1,2, \ldots$ and $\Phi\left(t_{1}, t_{2}, \ldots, t_{k}\right)$ a hereditary first-order formula such that $\neg \Phi$ is a Horn formula. Further, let $\vec{a}=\left(a_{1}, a_{2}, \ldots, a_{n}\right) \in \mathcal{R}^{(n)}, \vec{c}=\left(c_{1}, c_{2}, \ldots, c_{k}\right) \in \mathcal{R}^{(k)}$. Suppose that $\mathcal{R} \models \Phi(c)$ and for every maximal ideal $M$ of the Boolean ring $\mathcal{B}=\mathcal{B}(\mathcal{C})$, there exists a natural number $i=i(M)>0$ such that

$$
\mathcal{R}_{M} \models \Phi\left(\phi_{M}(\vec{c})\right) \Rightarrow \Theta_{i}\left(\phi_{M}(\vec{a})\right) .
$$

Then there exist a natural number $N$ and pairwise orthogonal idempotents $e_{1}, e_{2}, \ldots, e_{N}$ $\in \mathcal{B}$ such that $e_{1}+e_{2}+\cdots+e_{N}=1$ and $e_{i} R \models \Theta_{i}\left(e_{i} \vec{a}\right)$ for all $e_{i} \neq 0$ ", respectively.

Now, we are able to discuss our last result. 
Theorem 2.2. Let $\mathcal{R}$ be a 2-torsion free semiprime ring and $d$ be a nonzero derivation of $\mathcal{R}$ such that $\left.\left(\left[d\left([s, t]_{m}\right),[s, t]_{m}\right]\right)^{k}=d\left([s, t]_{m}\right),[s, t]_{m}\right]$ for all $s, t \in \mathcal{R}$. Further, let $\mathcal{A}=\mathcal{O}(\mathcal{R})$ is the orthogonal completion of $\mathcal{R}$ and $\mathcal{B}=\mathcal{B} \mathcal{C}$, where $\mathcal{C}$ is the extended centroid of $\mathcal{R}$. Then there exists a central idempotent element $e \in \mathcal{B}$ such that $d$

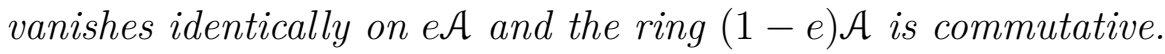

Proof. In view of our hypothesis, $\mathcal{R}$ satisfies

$$
\left(\left[d\left([s, t]_{m}\right),[s, t]_{m}\right]\right)^{k}=\left[d\left([s, t]_{m}\right),[s, t]_{m}\right] .
$$

Moreover, $\mathcal{U}$ satisfies $\left(\left[d\left([s, t]_{m}\right),[s, t]_{m}\right]\right)^{k}-\left[d\left([s, t]_{m}\right),[s, t]_{m}\right]=0$ for every $s, t \in \mathcal{U}$ (see [15]). By Remark 3.1.16 of [3], we conclude that $d(\mathcal{A}) \subseteq \mathcal{A}$ and $d(e)=0$ for every $e \in \mathcal{B}$. Therefore, $\mathcal{A}$ is an orthogonally complete $\Omega-\Delta$ - ring, where $\Omega=\{0,+,-, ., d\}$. Consider the formulas

$$
\begin{aligned}
\Phi & =\left\|\left(\left[d\left([s, t]_{m}\right),[s, t]_{m}\right]\right)^{k}-\left[d\left([s, t]_{m}\right),[s, t]_{m}\right]=0\right\|, \quad \text { for all } s, t, \\
\Theta_{1} & =\|s t=t s\|, \quad \text { for all } s, t \\
\Theta_{2} & =\|d(s)=0\|, \quad \text { for all } s .
\end{aligned}
$$

One can smartly verify that $\Phi$ is a hereditary first-order formula and $\neg \Phi, \Theta_{1}, \Theta_{2}$ are Horn formulas. Using Theorem 2.1, we can smartly verify that all the requirements of [3, Theorem 3.2. 18] are satisfied. Therefore, there exist two orthogonal idempotent $e_{1}$ and $e_{2}$ such that $e_{1}+e_{2}=1$ and if $e_{i} \neq 0$, then $e_{i} \mathcal{A} \models \Theta_{i}, i=1,2$. This completes the proof of the theorem.

\section{REFERENCES}

[1] M. Ashraf, M. A. Raza and S. Parry, Commutators having idempotent values with automorphisms in semi-prime rings, Math. Rep. (Bucur.) 20(70) (2018), 51-57.

[2] M. Ashraf and N. Rehman, On commutativity of rings with derivations, Results Math. 42 (2002), 3-8.

[3] K. I. Beidar, W. S. Martindale III and A. V. Mikhalev, Rings with Generalized Identities, Pure and Applied Mathematics, Marcel Dekker 196, New York, 1996.

[4] H. E. Bell and M. N. Daif, On derivations and commutativity in prime rings, Acta Math. Hung. 66 (1995), 337-343.

[5] C. L. Chuang, GPIs having coefficients in Utumi quotient rings, Proc. Amer. Math. Soc. 103 (1988), 723-728.

[6] M. N. Daif and H. E. Bell, Remarks on derivations on semiprime rings, Int. J. Math. Math. Sci. 15 (1992), 205-206.

[7] V. De Filippis, Generalized derivations in prime rings and noncommutative Banach algebras, Bull. Korean Math. Soc. 45 (2008), 621-629.

[8] V. De Filippis, M. A. Raza and N. Rehman, Commutators with idempotent values on multilinear polynomials in prime rings, Proc. Indian Acad. Sci. (Math. Sci.) 127(1) (2017), 91-98.

[9] A. Giambruno, J. Z. Goncalves, and A. Mandel, Rings with algebraic n-Engel elements, Comm. Algebra 22(5) (1994), 1685-1701.

[10] I. N. Herstein, A condition for the commutativity of the rings, Canad. J. Math. 9 (1957), 583-586.

[11] S. Huang, Derivations with Engel conditions in prime and semiprime rings, Czechoslovak Math. J. 61(136) (2011), 1135-1140. 
[12] N. Jacobson, Structure Theory for algebraic algebras of bounded degree, Ann. Math. 46(4) (1945), 695-707.

[13] V. K. Kharchenko, Differential identities of prime rings, Algebra Logic 17 (1979), 155-168.

[14] C. Lanski, An Engel condition with derivation, Proc. Amer. Math. Soc. 118 (1993), 731-734.

[15] T. K. Lee, Semiprime rings with differential identities, Bull. Inst. Math. Acad. Sin. (N>S.) 20 (1992), 27-38.

[16] W. S. Martindale III, Prime rings satisfying a generalized polynomial identity, J. Algebra 12 (1969), 576-584.

[17] M. A. Raza and N. Rehman, On prime and semiprime rings with generalized Derivations and non-commutative Banach algebras, Proc. Indian Acad. Sci. Math. Sci. 126(3) (2016), 389-398.

[18] M. A. Raza and N. Rehman, A note on an Engel condition with derivations in rings, Creat. Math. Inform. 26(1) (2017), 19-27.

[19] N. Rehman, M. A. Raza and T. Bano, On commutativity of rings with generalized derivations, J. Egyptian Math. Soc. 24(2) (2016), 151-155.

[20] L. Rowen, Polynomial Identities in Ring Theory, Pure and Applied Mathematics 84, Academic Press, New York, 1980.

${ }^{1}$ Department of Mathematics,

Faculty of Sciences and Ats-Rabigh,

King Abdulaziz University, Jeddah, SAudi Arabia

Email address: arifraza03@gmail.com

Email address: alhazmih@yahoo.com

${ }^{2}$ Department of Mathematics,

Aligarh Muslim University,

Aligarh-202002 InDiA

Email address: shakir50@rediffmail.com 